\title{
The long-term effect of tacrolimus on alkali burn-induced corneal neovascularization and inflammation surpasses that of anti-vascular endothelial growth factor
}

This article was published in the following Dove Press journal:

Drug Design, Development and Therapy

\section{Ling Chen* \\ Jing Zhong* \\ Saiqun Li \\ Weihua Li \\ Bowen Wang \\ Yuqing Deng \\ Jin Yuan}

State Key Laboratory of

Ophthalmology, Zhongshan

Ophthalmic Center, Sun Yat-sen

University, Guangzhou 510060, China

*These authors contributed equally to this work
Correspondence: Jin Yuan State Key Laboratory of Ophthalmology, Zhongshan Ophthalmic Center, Sun Yat-sen University, 54 South Xianlie Road, Guangzhou 510060, China $\mathrm{Tel} / \mathrm{fax}+86208733 \quad 1550$ Email yuanjincornea@।26.com
Purpose: To investigate the effect of tacrolimus in alkali burn-induced corneal neovascularization (NV) and inflammation and to compare with anti-vascular endothelial growth factor (anti-VEGF).

Methods: After corneal alkali-burn, 84 Wistar rats were randomly divided into three groups and received either saline solution or $0.05 \%$ tacrolimus $(0.5 \mathrm{mg} / \mathrm{mL})$ four times daily, or subconjunctival anti-VEGF injection $(0.5 \mathrm{mg} / 0.05 \mathrm{~mL})$. Corneal $\mathrm{NV}$, opacity and epithelial defects, the status of inflammation, and the levels of proinflammatory and angiogenic cytokines were assessed on Days 3, 7, 14 and 28 post-injury.

Results: Compared with the control, tacrolimus significantly reduced corneal NV on Days 7 , 14 and 28 post-injury, and anti-VEGF significantly reduced corneal NV at each assessment. Nevertheless, the tacrolimus group had significantly less corneal NV than the anti-VEGF group on Days 14 and 28. Furthermore, both tacrolimus and anti-VEGF significantly decreased the VEGF-A expression on Days 7 and 14, with no significant difference between the two groups. Moreover, corneal inflammatory response was alleviated, and corneal opacity and epithelial defects were significantly reduced by tacrolimus. Additionally, the expression of IL-1 $\beta$, IL-6, monocyte chemotactic protein-1, macrophage inflammatory protein- $1 \alpha$ and TGF- $\beta$ were significantly decreased by tacrolimus.

Conclusion: Our findings suggested that $0.05 \%$ tacrolimus suspension eye drops effectively reduced alkali burn-induced corneal NV and inflammation, with a better effect than subconjunctival anti-VEGF injections on Days 14 and 28.

Keywords: tacrolimus, anti-vascular endothelial growth factor, corneal alkali burn, corneal neovascularization, corneal inflammation

\section{Introduction}

Ocular alkali burn is a common trauma worldwide, particularly in developing countries. ${ }^{1}$ Caustic agents often cause extensive corneal damage, resulting in permanent visual impairment. ${ }^{2}$ Clinically, except for recurrent epithelial erosions, corneal ulceration and severe stromal inflammation, secondary corneal neovascularization (NV) is one of the most damaging conditions to sight. ${ }^{3}$ The cornea is an avascular and transparent tissue that acts as the refractive surface of the eye. ${ }^{4}$ After alkali burn, a large number of leukocytes and macrophages infiltrate the cornea; ${ }^{5}$ moreover, the levels of numerous cytokines, such as the inflammatory factors IL-1 $\beta$, IL-6 and vascular endothelial growth factor A (VEGF-A), are dramatically elevated in 
the alkali-burned cornea. ${ }^{6,7}$ The disruption of the balance between proangiogenic and antiangiogenic molecules will lead to NV of the cornea. ${ }^{8,9}$

Currently, the focus of corneal alkali burn treatment is anti-inflammatory therapies, such as steroids and NSAIDs. Furthermore, citrate, argon laser photocoagulation and photodynamic therapy are used to treat corneal inflammation and inflammatory NV induced by an alkali burn. ${ }^{10,11}$ Nevertheless, their clinical efficacy is limited, and undesirable side effects can occur. ${ }^{12,13}$ Anti-VEGF agents, such as bevacizumab and ranibizumab, effectively reduce NV by binding to VEGF-A and are the most effective methods used to inhibit NV at present; however, their role is confined to reducing inflammation, and their long-term use is expensive and leads to epithelial erosion or corneal thinning. ${ }^{14}$ Thus, further research exploring effective therapeutic protocols for alkali burninduced corneal inflammation and $\mathrm{NV}$ is imperative.

Tacrolimus (FK506), a macrolide molecule, suppresses inflammatory cascades by inhibiting the infiltration of lymphocytes and neutrophils, reducing free radical production from monocytes, and reducing the levels of related inflammatory cytokines IL-1, IL-6 and tumor necrosis factor. ${ }^{15,16}$ A previous study indicated that topical and subconjunctival FK506 application effectively reduced silver nitrate cauterization-induced corneal NV and the expression levels of VEGF, ${ }^{17}$ but the study only detected the effects for a short term (7 days). Moreover, tacrolimus demonstrated comparable effects to subconjunctival bevacizumab injection on corneal stromal suture-induced corneal NV during a short-term study (7 days). ${ }^{18}$ In addition, FK506 has also proven effective in the treatment of corneal graft rejection, inflammatory conjunctival and corneal diseases, uveitis, and ocular graft-versus-host disease. ${ }^{19,20}$ Our previous study indicated that $0.05 \%(0.5 \mathrm{mg} / \mathrm{mL})$ FK506 suspension eye drops was a suitable candidate for clinical application in ophthalmology. ${ }^{21}$ And, we also demonstrated that up to $0.1 \%$ FK506 administered topically, four times daily, is safe for the rabbit eye. ${ }^{22}$ At present, $0.05 \%$ FK506 has been widely used in clinics to relieve rejection after keratoplasty, and so on. However, its therapeutic effect and mechanism in alkali burn-induced corneal NV and inflammation remains unknown.

In this study, an alkali burn-induced rat model was employed to explore the effect and mechanism of tacrolimus on corneal alkali burn and to compare the long-term effect of tacrolimus and anti-VEGF on alkali burn-induced corneal $\mathrm{NV}$ and inflammation.

\section{Materials and methods}

\section{Animals}

Wistar rats (specific-pathogen-free grade, 6-8 weeks old, 200-250 g, with equal numbers of males and females) were obtained from the Southern Medical University animal breeding center, Guangzhou, China. The Institutional Animal Care and Use Committee of Zhongshan Ophthalmic Center, Sun Yat-sen University, Guangzhou, China approved the study protocol. All animal experiments complied with the National Institutes of Health Guidelines for the Care and Use of Laboratory Animals (NIH Publications No 8023, revised 1978). The right eye was experimental eye, and the left eye was intact.

\section{Corneal alkali burn model}

The alkali burn model was improved according to the previous method. ${ }^{9}$ The rats were anesthetized via an intraperitoneal injection of ketamine hydrochloride and xylazine combination (94 mg/kg body weight), and the topical anesthetic lidocaine was used. A total of $12 \mu \mathrm{L}$ of $1 \mathrm{~mol} / \mathrm{L}$ sodium hydroxide was added to a circular piece of Whatman \#3 filter paper ( $5 \mathrm{~mm}$ in diameter), which was then pressed onto the central cornea of the right eye for 30 seconds. The corneal surface was then carefully rinsed with $60 \mathrm{~mL}$ of physiologic saline solution. After the alkali burn was induced, the rats $(n=84)$ were divided into three groups. The control group $(n=28)$ was treated with a topical instillation of saline solution eye drops four times daily until the time the animals were euthanized. The FK506 group ( $\mathrm{n}=28$ ) was given topical $0.05 \%$ FK506 $(0.5 \mathrm{mg} / \mathrm{mL})$ eye drops (Zhongshan Ophthalmic Center, Guangzhou, China) four times daily until the time animals were euthanized. The anti-VEGF group $(\mathrm{n}=28)$ received a single dose of $0.5 \mathrm{mg}(0.05 \mathrm{~mL})$ anti-rat VEGF (Abcam, Cambridge, UK) via a subconjunctival single injection immediately after the corneal alkali burn. ${ }^{23}$

\section{Evaluation of corneal opacity}

The animals' condition was monitored once per day. On Days 3, 7, 14 and 28 post-injury, seven rats from each group were randomly chosen for corneal opacity evaluation. Two researchers, who were blind to the study groups, independently examined corneal opacity under a slit lamp and obtained photographs with a slit lamp camera system (Carl Zeiss, Inc., Jena, Germany). Corneal opacity was graded according to Sonoda and Streilein. ${ }^{24}$

\section{Quantification of corneal NV}

On Days 3, 7, 14 and 28 post-injury, seven rats from each group were randomly chosen for corneal NV assessment. 
Images of the corneas were obtained, and the area of NV was measured automatically by ImageJ software (http:// imagej.nih.gov/ij/).

\section{Measurement of corneal epithelial damage}

Five microliter of a $0.1 \%$ fluorescein sodium solution was instilled in the rats' eyes. After 30 seconds, the eyes were examined with cobalt blue light under a slit lamp and were photographed with a slit lamp camera system. ImageJ software was used to measure the percentage of defect area.

\section{Ink perfusion}

Two rats from each group underwent ink perfusion on Day 28 as previously described. ${ }^{25}$ The rats were deeply anesthetized, and the thorax cavity was surgically exposed to reach the heart. A syringe needle (diameter $=1.6 \mathrm{~mm}$ ) was inserted into the left ventricle, and a small slit was cut in the right atrium. The inferior vena artery was occluded with a vascular clamp, and saline solution was perfused through the aorta until no blood flowed out of the right atrium. Then, $10 \mathrm{~mL}$ of $10 \%$ India ink was perfused in the same manner. Subsequently, the rats were euthanized, and the eyes were enucleated and fixed in a $4 \%$ paraformaldehyde solution overnight. The cornea was carefully dissected under a biomicroscope for observation and photography with a microscope camera system (Leica Camera AG, Wetzlar, Germany) the next day.

\section{Histologic examination}

H\&E staining was performed on two randomly selected rats from each group on Days 3, 7 and 14. The rats were euthanized, and the right eye was enucleated, then an incision was made outside of the cornea. Then, the eyes were fixed in a $4 \%$ paraformaldehyde solution overnight. The cornea was dissected and washed with PBS and then embedded in paraffin the next day. Subsequently, paraffin blocks were sliced into $\sim 3 \mu \mathrm{m}$ sections and stained with H\&E.

\section{Quantitative Real-Time PCR}

Gene and protein expression levels were examined in corneas of five randomly selected rats from each group on Days 3, 7, 14 and 28 post-injury. Each cornea was divided into two slices, and total RNA was extracted from one-half of each cornea. The corneas were cut into small pieces and homogenized in TRIzol ${ }^{\mathrm{TM}}$ (Invitrogen, Carlsbad, CA, USA) on ice using an ultrasonic crusher. Total RNA was extracted according to the manufacturer's instructions. Then, $100 \mathrm{ng}$ of RNA was reverse-transcribed into cDNA using Oligo (dT) and a PrimeScript ${ }^{\mathrm{TM}}$ RT Reagent Kit (Takara Bio Inc., Kusatsu, Japan). The mRNA levels of VEGF-A, IL-1 $\beta$, IL-6, MCP-1, MIP- $1 \alpha$ and TGF- $\beta$ were quantified via real-time PCR with SYBR ${ }^{\text {TM }}$ Green (Kapa Biosystems, Wilmington, MA, USA). The assays were conducted in triplicate, and the relative expression levels were determined by normalizing the expression of each target gene to GAPDH using the $2^{-\Delta \Delta \mathrm{Ct}}$ method. In this study, Primer3 (http://primer3.ut.ee/) was used for designing primers, and the specificity of the primers was verified by NCBI Primer-BLAST (http://blast. ncbi.nlm.nih.gov/Blast.cgi). Primer sequences for qRT-

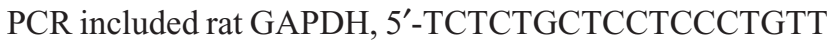
CTA-3' and 5'-AAGAGAAGGCAGCCCTGG TAA-3'; rat VEGF-A, 5'-CCAAAGCCAGCACATAGGAGA-3' and 5'-TCTGCGGA TCTTGGACAAACA-3'; rat IL-1 $\beta$, 5'-TGAAGCAGCTATGGCAACTGT-3' and 5'-CATCT GGACAGCCCAAGTCAA-3'; rat IL-6, 5'-TCACAGA GGATACCACCCACA-3' and 5'-CAGTGCATCA TCGCTGTTCAT-3'; rat MCP-1, 5' -TGTTCACAGTTGCT GCCTGTA-3' and 5'-AGTTCTCCAGCCGACTCATTG-3'; rat MIP- $1 \alpha, 5^{\prime}$-TGCTG TTCTTCTCTGCACCAT-3' and 5'-TCGTGGAATTTGCCGTCCATA-3'; and rat TGF- $\beta$, 5'-CAGTGGCTGAACCAAGGAGA-3' and 5'-GGAAGGGTCGGTTCAT GTCA-3'.

\section{ELISA}

The other half of each cornea was used for protein examination. Briefly, the corneas were cut into small pieces and homogenized in a tissue extraction reagent on ice using an ultrasonic crusher. Total protein concentrations were measured using a BCA protein assay kit (Bio-Rad, Hercules, CA, USA). Total protein $(20 \mu \mathrm{g})$ from each sample was used for measurements of VEGF-A, IL-1 $\beta$, IL-6, MCP-1, MIP- $1 \alpha$ and TGF- $\beta$ with ELISA kits (R\&D Systems ${ }^{\circledR}$, Minneapolis, MN, USA) according to the manufacturer's instructions. The ELISA experiments were performed in triplicate.

\section{Statistics}

The statistical analyses were performed using SPSS version 21.0 (IBM, Armonk, NY, USA). Differences in the areas of NV, corneal opacity grade, corneal epithelial deficiency, expression levels of VEGF-A, and inflammatory and chemotactic cytokines were analyzed via one-way ANOVA with the least significant difference post hoc test. Significant differences were defined as $P<0.05$. 


\section{Results}

FK506 reduced alkali burn-induced corneal NV and opacity

Representative slit lamp images show that the area of NV markedly increased over time in the control group, with increasing mean areas of $4.45 \mathrm{~mm}^{2}, 8.54 \mathrm{~mm}^{2}, 26.74 \mathrm{~mm}^{2}$ and $28.01 \mathrm{~mm}^{2}$ on Days 3, 7, 14 and 28 after alkali burn, respectively, while less NV was observed in the FK506 and anti-VEGF groups at every checkpoint. Moreover, corneal opacity was markedly decreased over time in the FK506 and anti-VEGF groups, but not in the control group (Figure 1A). In addition, FK506 significantly reduced corneal NV area on
A
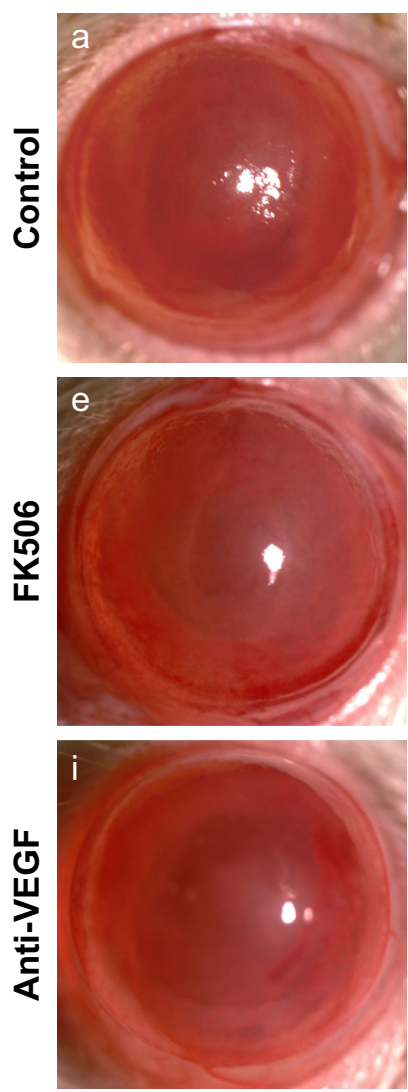

B

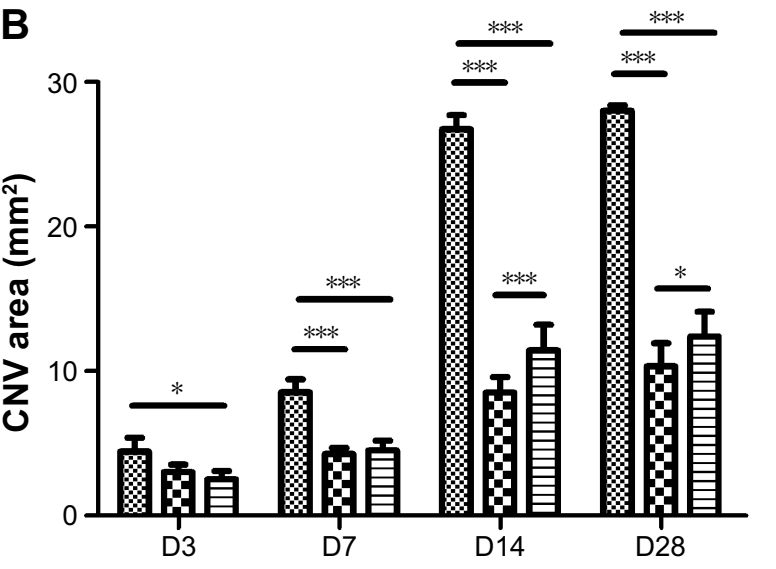

D7
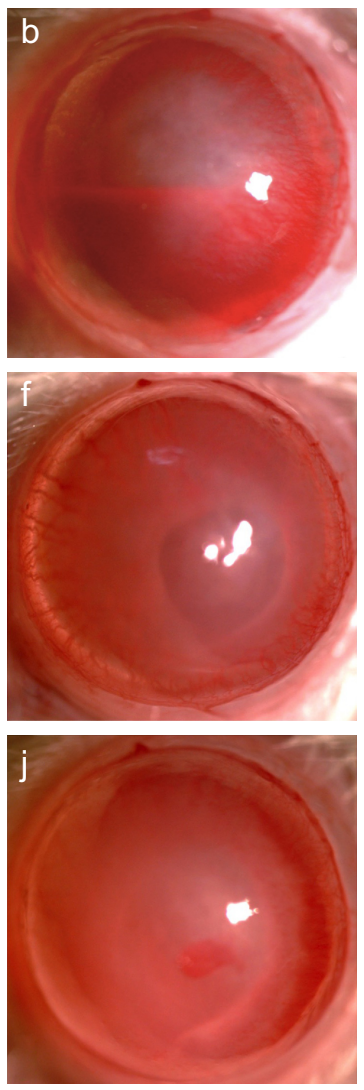

C

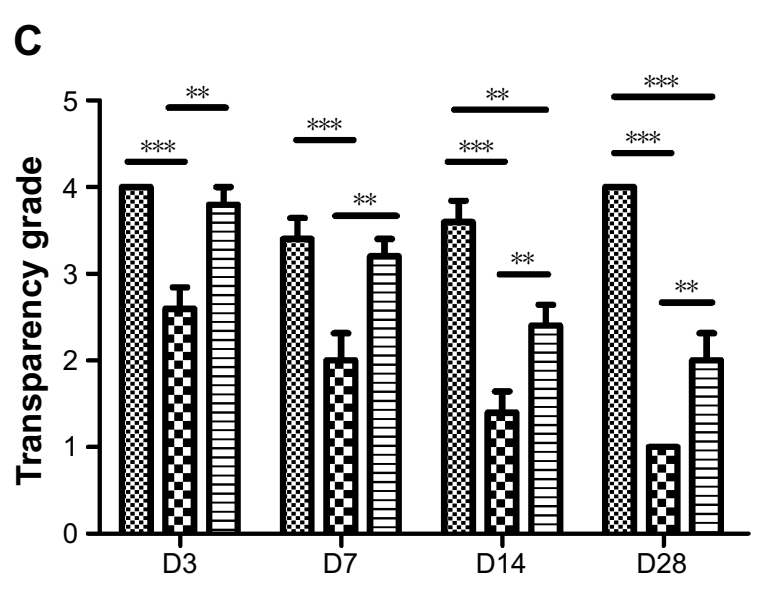

D28
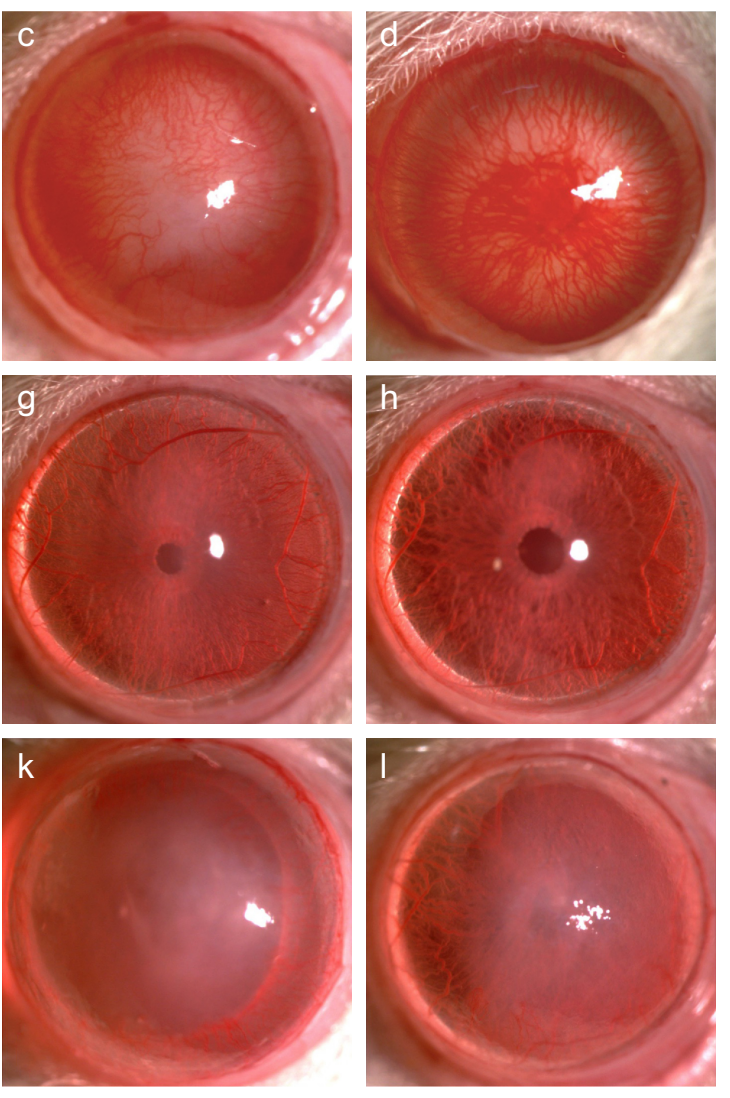

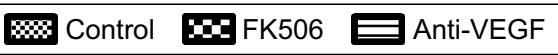

Figure I FK506 reduced NV and corneal opacity in a corneal alkali burn model.

Notes: (A) Representative ocular surface images of rats in the control group (a-d), rats in the FK506 group (e-h) and rats in the anti-VEGF group (i-I) on Days 3, 7, I4 and 28 post-injury. Magnification $\times$ I0. (B) Time-dependent changes in corneal NV area among the three groups on Days 3, 7, I4 and 28 post-injury. (C) Time-dependent changes in corneal opacity grades among the three groups on Days 3, 7, I4 and 28 post-injury. (Data are presented as the mean $\pm S E M, n=7$. $* P<0.05, * * P<0.01, * * * P<0.001$ ). Abbreviations: Anti-VEGF, anti-vascular endothelial growth factor; CNV, corneal neovascularization; FK506, tacrolimus; NV, neovascularization. 
Days 7, 14 and 28 post-injury (all $P<0.001$, ANOVA) but not on Day $3(P=0.058)$, while anti-VEGF significantly reduced corneal NV area on Days $3(P<0.05), 7,14$ and $28(P<0.001)$. Moreover, the NV area in the FK506 group was significantly smaller than that in the anti-VEGF group on Days 14 and 28 post-injury ( $P<0.001$ and $P<0.05$, respectively) (Figure 1B). Additionally, compared with control and anti-VEGF, FK506 significantly reduced corneal opacity from Day 3 to Day 28 (all $P<0.01$ ). Furthermore, compared with the control, antiVEGF significantly reduced corneal opacity on Days 14 and 28 ( $P<0.01$ and $P<0.001$, respectively) (Figure 1C).

\section{FK506 reduced the expression levels of VEGF-A in a corneal alkali burn model}

The mRNA (Figure 2A) and protein (Figure 2B) levels of VEGF-A visibly increased after alkali burn, reached a peak on Day 7 post-injury. The mRNA and protein levels of VEGF-A in the FK506 group were significantly lower than those in the control group on Days 7 and 14 (all $P<0.05$ ) but not on Day 3 ( $P=0.387$ ), while the VEGF-A expression level in the anti-VEGF group was significantly reduced on Days 3, 7 and 14 (all $P<0.05$ ) compared with control group. No significant differences were observed between the FK506 and anti-VEGF groups on all checkpoints. In addition, ink perfusion was used on Day 28 in each group to explore whole mounts of neovascularized corneas, which revealed new vessels with black staining. New vessels extended into every fraction of the cornea in the control group, while there were fewer new vessels in the anti-VEGF group. The FK506 group exhibited the fewest new vessels, which were distributed only at the periphery of the cornea (Figure 2C). The NV distribution revealed by ink perfusion was consistent with the NV area calculation results shown in Figure 1B.

\section{FK506 accelerated corneal wound healing in a corneal alkali burn model}

To explore the role of FK506 in corneal wound healing, we examined corneal epithelial defects with fluorescein staining
A

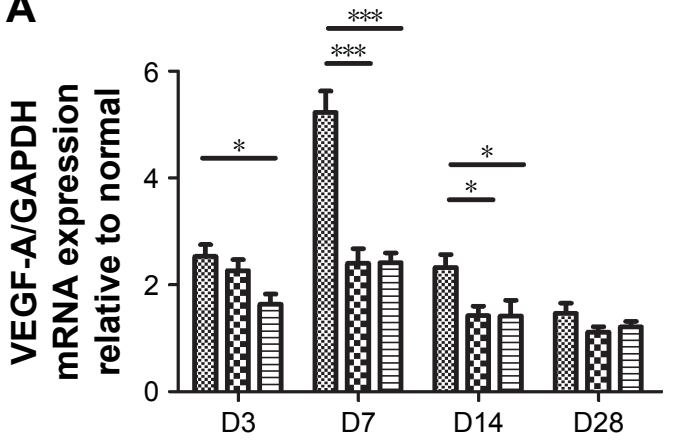

B

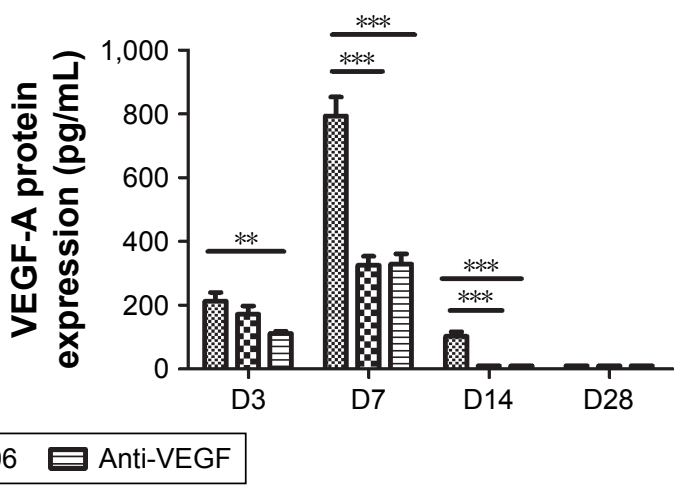

C

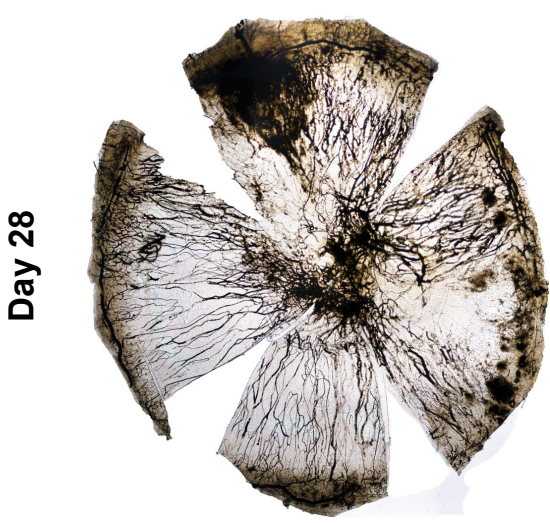

FK506

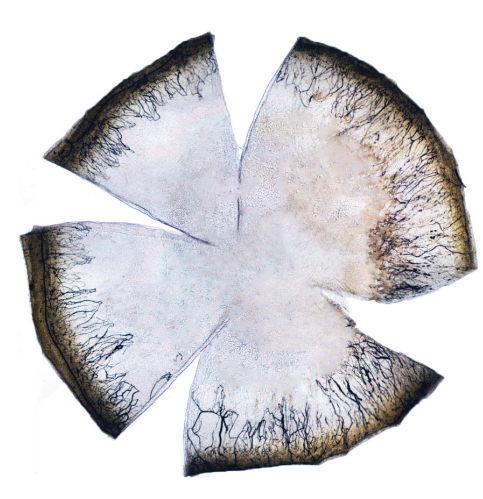

Anti-VEGF

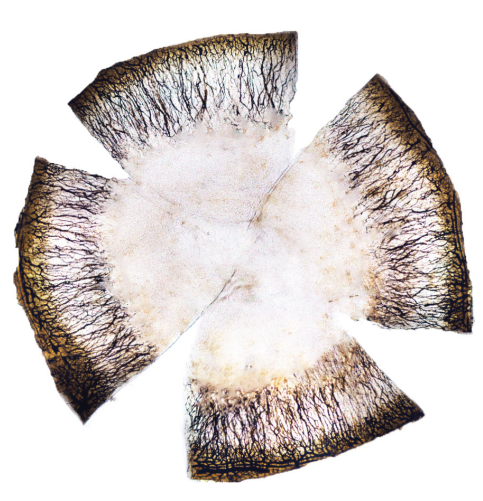

Figure 2 FK506 inhibited NV by reducing the expression levels of VEGF-A in a corneal alkali burn model.

Notes: (A) VEGF mRNA expression in the control group, the FK506 group and the anti-VEGF group on Days 3, 7, 14 and 28 post-injury. (B) VEGF-A protein expression in the three groups on Days 3, 7, 14 and 28 post-injury. (C) Representative ink perfusion images of whole mounts of rat corneas treated with saline solution, FK506 or antiVEGF on Day 28 post-injury. (Data are presented as the mean \pm SEM, $n=5 . * P<0.05, * * p<0.01, * * * p<0.00$ I). Magnification $\times 40$.

Abbreviations: Anti-VEGF, vascular endothelial growth factor; FK506, tacrolimus; NV, neovascularization. 

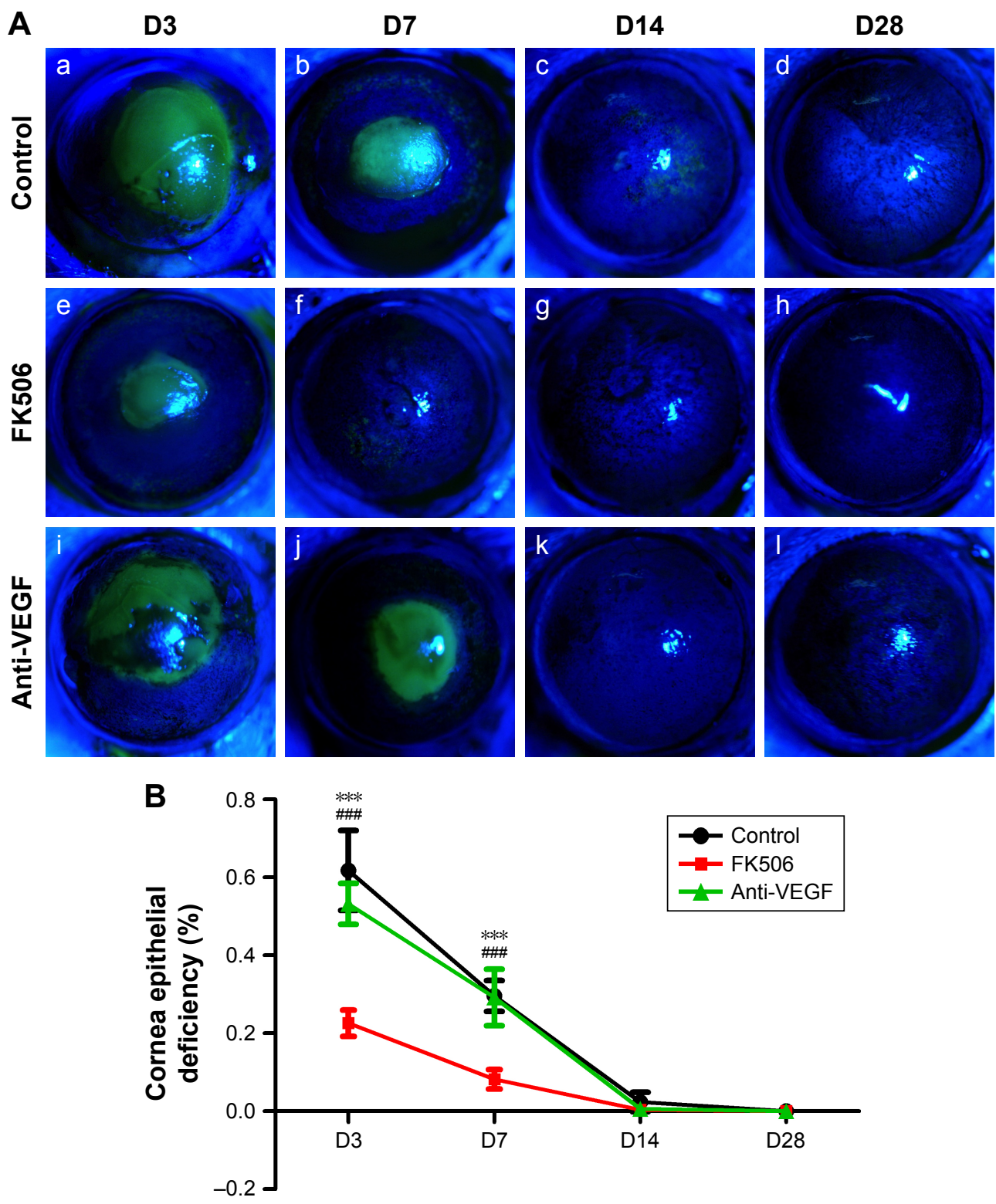

Figure 3 FK506 promoted corneal wound healing in a corneal alkali burn model.

Notes: (A) Representative ocular surface fluorescein staining images of rats in the control group (a-d), rats in the FK506 group (e-h) and rats in the anti-VEGF group (i-l) on Days 3, 7, 14 and 28 post-injury. Magnification $\times 10$. (B) Time-dependent changes in the percentage of corneal epithelial defect area in the three groups on Days 3 , 7, I4 and 28 post-injury. (Data are presented as the mean \pm SEM, $n=7$. $* * * P<0.00$ I for the FK506 group vs the control group; ${ }^{\#} P<0.00$ I for the FK506 group vs the anti-VEGF group). Abbreviations: Anti-VEGF, vascular endothelial growth factor; FK506, tacrolimus.

from Day 3 to 28 in the different groups. Figure $3 \mathrm{~A}$ shows the representative fluorescein staining images. On Day 3 , the corneal epithelial deficiency rate was $\sim 62 \%$ in the control group, $23 \%$ in the FK506 group and 53\% in the anti-VEGF group. On Day 7, the corneal epithelium deficiency rate was $\sim 30 \%, 8 \%$ and $29 \%$ in the three groups, respectively. On Days 14 and 28, the corneal epithelium was almost healed in the three groups. Furthermore, the corneal epithelium deficiency rate in the FK506 group was significantly lower than that in the control and anti-VEGF groups on Days 3 and 7 (all $P<0.001$ ), while there was no significant difference between the control and anti-VEGF groups. Additionally, there was no significant difference in the corneal epithelium deficiency rate among the three groups on Days 14 and 28 (Figure 3B).

\section{FK506 alleviated cellular infiltration in a corneal alkali burn model}

The representative $\mathrm{H} \& \mathrm{E}$ staining images (Figure $4 \mathrm{~A}-\mathrm{C}$ ) show that numerous inflammatory cells infiltrated the injured cornea on Days 3, 7 and 14, and obvious NV cavities were observed on Day 14 in the control group. Furthermore, FK506 noticeably decreased not only the number of the infiltrating inflammatory cells but also the number of $\mathrm{NV}$ 


\section{Day 3}
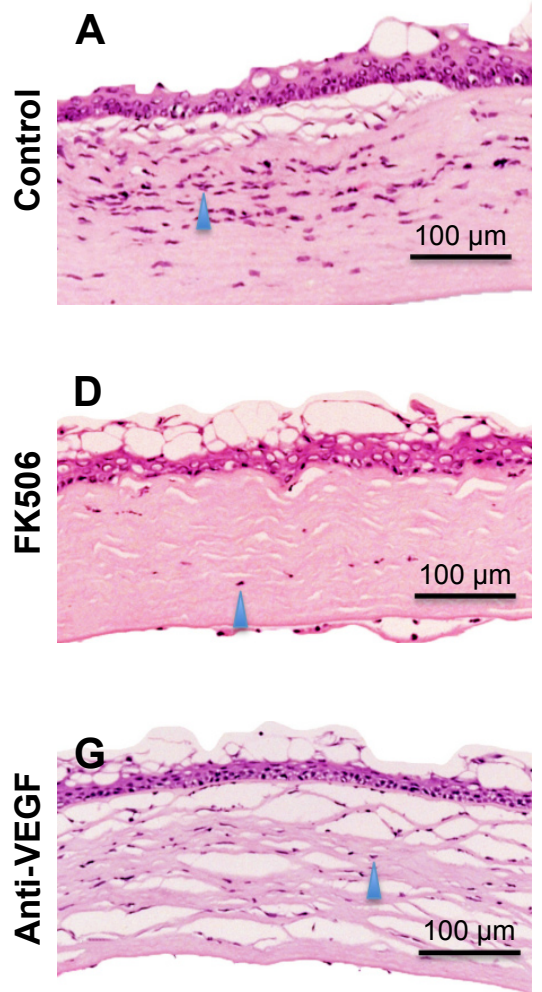

Day 7
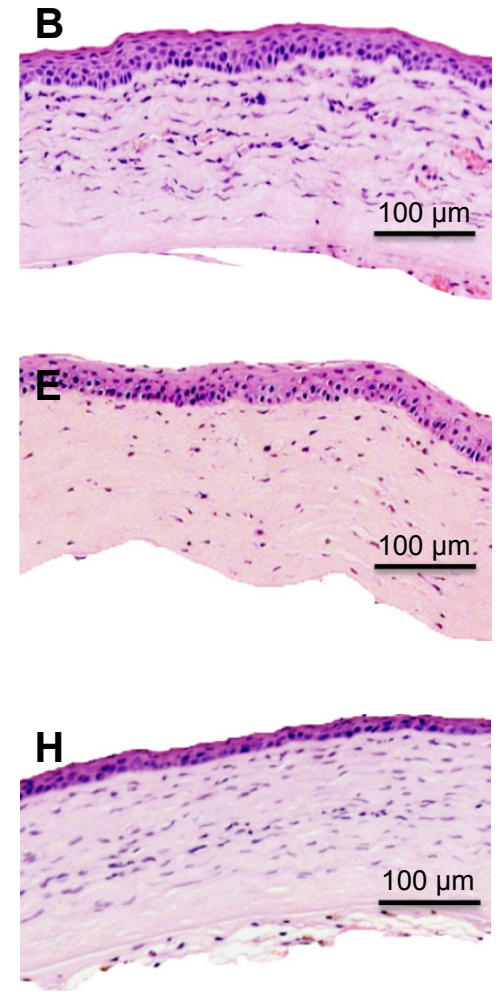

Day 14
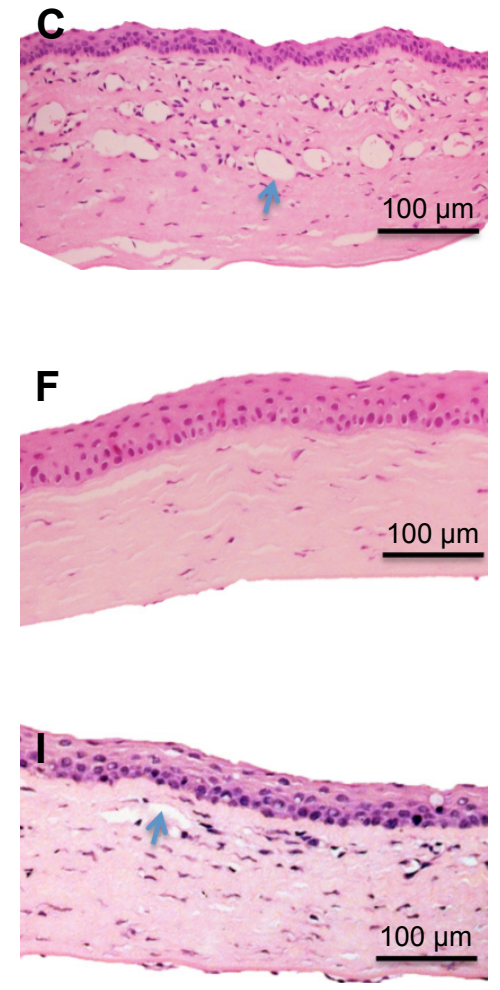

Figure 4 FK506 suppressed inflammatory cell infiltration in a corneal alkali burn model.

Notes: Representative H\&E staining images of rats in the control group (A-C), rats in the FK506 group (D-F) and rats in the anti-VEGF group (G-I) on Days 3, 7 and I4 post-injury. FK506 obviously suppressed inflammatory cell infiltration in alkali-burned corneas compared with the control group and the anti-VEGF group (blue triangles); furthermore, NV cavities were obviously decreased by FK506 and anti-VEGF (blue arrows).

Abbreviations: Anti-VEGF, vascular endothelial growth factor; FK506, tacrolimus; NV, neovascularization.

cavities (Figure 4D-F). In addition, the number of infiltrating inflammatory cells was slightly decreased by anti-VEGF on Days 3, 7 and 14. Furthermore, the number of NV cavities in the anti-VEGF group was markedly lower than that in the control group on Day 14 (Figure 4G-I).

\section{FK506 reduced the expression levels of inflammatory and chemotactic cytokines in a corneal alkali burn model}

To explore the mechanism of FK506 in the alkali-burned cornea, we evaluated the expression levels of inflammatory cytokines (IL-1 $\beta$ and IL-6), chemotactic cytokines (MCP-1 and MIP- $1 \alpha$ ) and TGF- $\beta$ from Day 3 to Day 28 post-injury in each group (Figure 5A-J). The mRNA and protein levels of IL-1 $\beta$, MCP-1 and MIP- $1 \alpha$ were noticeably elevated in the control group and peaked on Day 3. Furthermore, the mRNA and protein levels of IL- 6 and TGF- $\beta$ were notably elevated in the control group and peaked on Day 7. In addition, compared with control, FK506 significantly decreased the mRNA levels of IL-1 $\beta$ (all $P<0.01$ ), IL-6 (all $P<0.001$ ), MCP-1 (all $P<0.01$ ), MIP- $1 \alpha$ (all $P<0.01$ ) and TGF- $\beta$ (all $P<0.01$ ) on Days 3, 7 and 14, as well as the mRNA levels of IL- $1 \beta$ and IL-6 on Day 28 (both $P<0.05$ ) (Figure 5A-E). Moreover, compared with control, anti-VEGF significantly decreased the mRNA levels of IL-1 $\beta$, IL-6, MCP-1, MIP- $1 \alpha$ and TGF- $\beta$ (all $P<0.05$ ) on Days 3 and 7 (Figure 5A-E). The mRNA levels of these factors in the FK506 group were significantly decreased on Days 3, 7 and 14 compared with those in the anti-VEGF group. Additionally, the protein levels of IL-1 $\beta$, IL-6, MCP-1 and MIP- $1 \alpha$ on Days 3, 7 and 14 and TGF- $\beta$ on Days 3 and 7 were significantly decreased in the FK506 group compared with those in the control group (all $P<0.05$ ). Compared with the control, anti-VEGF also significantly decreased the protein levels of IL-1 $\beta$, IL-6, MCP-1 and TGF- $\beta$ on Days 3 and 7, as well as MIP- $1 \alpha$ on Day 3 (all $P<0.05$ ) but exhibited higher levels than the FK506 group (Figure 5F-J).

\section{Discussion}

Alkali injury is one of the most devastating injuries to the eye and causes severe corneal damage. ${ }^{1}$ Controlling inflammation is critical in the treatment of corneal alkali burns. Anti-inflammatory medication, including steroids, NSAIDs and cyclosporine A are administered, but do not 

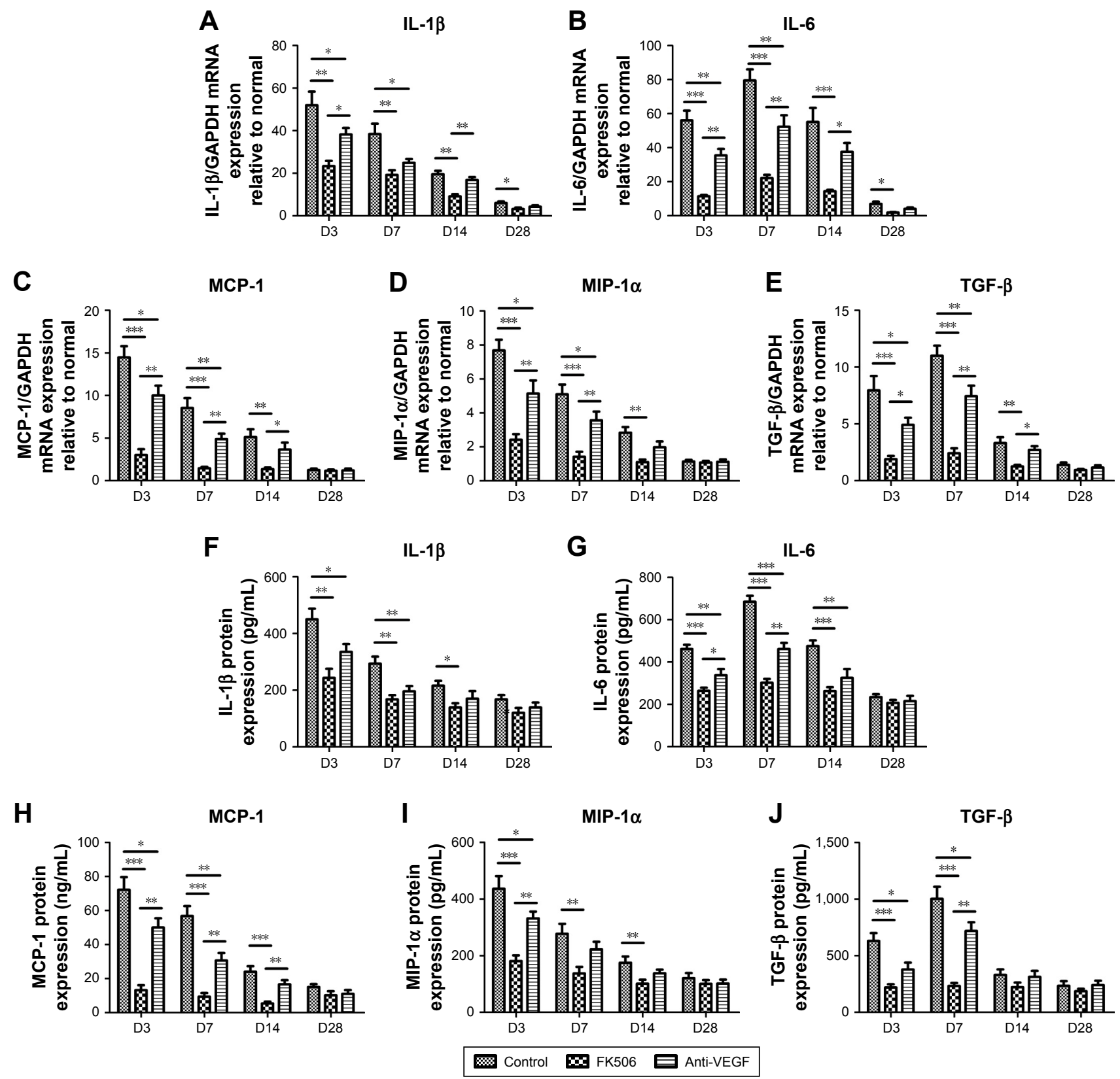

Figure 5 FK506 reduced increases in the expression of inflammatory cytokines and chemokines in a corneal alkali burn model.

Notes: The mRNA expression levels of IL-I $\beta$ (A), IL-6 (B), MCP-I (C), MIP-I $\alpha$ (D) and TGF- $\beta$ (E) in the control group, the FK506 group and the anti-VEGF group on Days 3, 7, 14 and 28 post-injury. The protein expression levels of IL-I $\beta$ (F), IL-6 (G), MCP-I (H), MIP-I $\alpha$ (I) and TGF- $\beta$ (J) in the control group, the FK506 group and the anti-VEGF group on Days $3,7,14$ and 28 post-injury. (Data are presented as the mean $\pm S E M, n=5$. $* P<0.05, * * P<0.01, * * * P<0.001$ ).

Abbreviations: Anti-VEGF, vascular endothelial growth factor; FK506, tacrolimus.

produce optimal clinical effects. ${ }^{26}$ Furthermore, the alkaliburned cornea is in a dysregulated chronic inflammatory state, which results in the formation of NV, a major sightthreatening complication in corneal alkali injury. ${ }^{9}$ AntiVEGF has limited efficacy in the treatment of inflammatory $\mathrm{NV}$, and corneal transplantation to replace scarred corneal tissue following alkali injury is complicated by a higher incidence of rejection than that of transplantation following other injuries. ${ }^{27}$ Overall, current treatment outcomes in alkali-burned corneas are generally unsatisfactory, and the development of more effective and safer treatments is needed.

Tacrolimus (FK506), a potent immunosuppressive macrolide, acts primarily by suppressing both B-cell and T-cell activation and proliferation. ${ }^{15,28}$ Clinically, topical FK506 is effective in ocular surface diseases, such as superior limbic keratoconjunctivitis, atopic keratoconjunctivitis and penetrating keratoplasty. ${ }^{29,30}$ Although the role of FK506 in many ocular surface diseases has been explored, little is known about its role and mechanism in corneal alkali burns. 
The present study explored the effect and mechanism of tacrolimus on corneal alkali burn and compared the long-term effect of tacrolimus and anti-VEGF on alkali burn-induced corneal NV and inflammation.

In alkali-burned corneas, inflammation is the critical and first stage in which immune cells, such as neutrophils, lymphocytes and monocytes, are activated and recruited by chemokines, such as MCP-1 and MIP-1 $\alpha$, to induce downstream pathways. ${ }^{31,32}$ In the present study, we assessed the effects of FK506 on the status of inflammation in alkali injury cornea. Our study showed that the mRNA and protein levels of the chemokines MCP-1 and MIP-1 $\alpha$ were increased and significantly inhibited by FK506 in alkali burned cornea; furthermore, FK506 markedly reduced the number of infiltrating inflammatory cells, as shown by H\&E staining, which indicated the role of FK506 in alleviating inflammation by reducing the infiltration and activation of inflammatory cells after a corneal alkali burn. In addition, the inflammatory cytokines IL-1 $\beta$ and IL-6 were significantly inhibited, which confirmed the effects of FK506 in inhibiting the activation and release of inflammatory cytokines. Additionally, a single subconjunctival injection of anti-VEGF reduced the number of infiltrating inflammatory cells and the expression levels of the chemokines MCP-1 and MIP- $1 \alpha$ along with the inflammatory cytokines IL-1 $\beta$ and IL-6. Consistent with previous research findings, anti-VEGF inhibited macrophage recruitment by reducing $\mathrm{NV}$ formation. ${ }^{18}$ Nevertheless, our study demonstrated that the effect of FK506 on alkali burninduced corneal inflammation significantly surpassed that of anti-VEGF.

Alkali burn-induced NV, a common and serious complication, is closely related to inflammation. In the normal mature ocular vascular system, angiogenesis-stimulating factors and angiogenesis inhibitors exist in a homeostatic balance. Under the pathologic conditions of alkali injury, the balance between angiogenesis stimulators and inhibitors is disrupted, leading to the formation of pathologic new vessels. ${ }^{33,34}$ VEGF-A is the most important factor that regulates angiogenesis, while inflammatory cytokines, such as IL-1 $\beta$ and IL-6, are also important factors that lead to NV.,35

Our study showed that FK506 significantly reduced alkali burn-induced corneal NV on Days 7, 14 and 28 post-injury, but not on Day 3. However, corneal NV area was significantly reduced by anti-VEGF at all assessment points, which means that anti-VEGF works faster than FK506 on inhibiting corneal NV. Indeed, the mRNA and protein expression levels of VEGF-A were significantly reduced by anti-VEGF but not by FK506 on Day 3. This might be explained by the difference of mechanisms between anti-VEGF and FK506 on corneal
NV. Furthermore, FK506 and anti-VEGF almost equally inhibited NV on Day 7, but FK506 was superior to anti-VEGF on Days 14 and 28. A previous study demonstrated that $0.1 \%$ tacrolimus eye drops, $0.5 \%$ tacrolimus subconjunctival injection and subconjunctival bevacizumab injection had comparable effects on stromal suture-induced corneal NV during a short-term observation ( 7 days),${ }^{18}$ which is consistent with our findings. Our study further demonstrated that the long-term effect of FK506 on alkali burn-induced corneal NV was better than that of the anti-VEGF subconjunctival single injection. A previous study also demonstrated that systemic $(0.3 \mathrm{mg} / \mathrm{kg})$ and topical $(0.03 \%)$ administration of tacrolimus might be beneficial in the prevention of corneal NV in rats induced by silver nitrate cauterization because of its effect on VEGF. ${ }^{17}$ Our study demonstrated that there was no significant difference for the expression of VEGF-A between the FK506 and anti-VEGF groups on Days 7, 14 and 28, but the NV area in FK506 group was significantly less than the anti-VEGF group on Days 14 and 28. The mechanism of FK506 decreasing VEGF-A expression was still unclear, and we speculated that FK506 reduced NV formation not only by reducing the VEGF-A expression but also by inhibiting inflammatory response, with the latter maybe playing a major role.

The corneal wound healing process involves complex interactions, which were negatively correlated with the inflammatory response in our previous study. ${ }^{36}$ FK506 attenuated the inflammatory response and reduced the inflammatory cytokines IL- $1 \beta$ and IL-6, which could accelerate epithelial migration. In addition, we assessed the levels of TGF- $\beta$, which plays important roles in regulating cell growth, epithelial-mesenchymal transition and tissue fibrosis. ${ }^{37}$ FK506 abrogates TGF- $\beta$-induced type I collagen production in normal human fibroblasts. ${ }^{38}$ The mRNA and protein expression levels of TGF- $\beta$ were markedly increased in alkali-burned rat corneas but were significantly inhibited by FK506. Hence, it indicated that FK506 might promote corneal epithelial healing and reduce the degree of corneal opacity by attenuating the inflammatory response and downregulating the levels of inflammatory cytokines and TGF- $\beta$ in an alkali burn rat model.

\section{Conclusion}

FK506 significantly reduced alkali burn-induced corneal NV and inflammation. The effect of FK506 on alkali burninduced corneal NV is slower than that of anti-VEGF, but with an effect surpassing that of anti-VEGF on Days 14 and 28. In addition, FK506 effectively reduced corneal opacity and facilitated corneal epithelial wound healing in the corneal alkali burn model. This work provides a better understanding 
of the role of FK506 in the treatment of corneal alkali burns and indicates promising new therapeutic directions.

\section{Acknowledgment}

This investigation was supported by grants from the National Key R\&D Program of China (grant number 2017 YFC0112400) and the National Natural Science Foundation of China (grant number 81270972).

\section{Disclosure}

The authors report no conflicts of interest in this work.

\section{References}

1. Singh P, Tyagi M, Kumar Y, Gupta KK, Sharma PD. Ocular chemical injuries and their management. Oman J Ophthalmol. 2013;6(2): 83-86.

2. Brodovsky SC, McCarty CA, Snibson G, et al. Management of alkali burns: an 11-year retrospective review. Ophthalmology. 2000;107(10): $1829-1835$.

3. Ye J, Yao K, Kim JC. Mesenchymal stem cell transplantation in a rabbit corneal alkali burn model: engraftment and involvement in wound healing. Eye. 2006;20(4):482-490.

4. Delmonte DW, Kim T. Anatomy and physiology of the cornea. J Cataract Refract Surg. 2011;37(3):588-598.

5. Wagoner MD. Chemical injuries of the eye: current concepts in pathophysiology and therapy. Surv Ophthalmol. 1997;41(4):275-313.

6. Sotozono C, He J, Matsumoto Y, Kita M, Imanishi J, Kinoshita S. Cytokine expression in the alkali-burned cornea. Curr Eye Res. 1997; 16(7):670-676.

7. Liu G, Wu H, Lu P, Zhang X. Interleukin (IL)-17A Promotes angiogenesis in an experimental corneal neovascularization model. Curr Eye Res. 2017;42(3):368-379.

8. Tzeng HE, Tsai CH, Chang ZL, et al. Interleukin-6 induces vascular endothelial growth factor expression and promotes angiogenesis through apoptosis signal-regulating kinase 1 in human osteosarcoma. Biochem Pharmacol. 2013;85(4):531-540.

9. Shin YJ, Hyon JY, Choi WS, et al. Chemical injury-induced corneal opacity and neovascularization reduced by rapamycin via TGF- $\beta 1 /$ ERK pathways regulation. Invest Ophthalmol Vis Sci. 2013;54(7): 4452-4458.

10. Shakiba Y, Mansouri K, Arshadi D, Rezaei N. Corneal neovascularization: molecular events and therapeutic options. Recent Pat Inflamm Allergy Drug Discov. 2009;3(3):221-231.

11. Gupta D, Illingworth C. Treatments for corneal neovascularization: a review. Cornea. 2011;30(8):927-938.

12. Mirabelli P, Peebo BB, Xeroudaki M, Koulikovska M, Lagali N. Early effects of dexamethasone and anti-VEGF therapy in an inflammatory corneal neovascularization model. Exp Eye Res. 2014;125:118-127.

13. Rho DS. Three cases of corneal melting after instillation of a new nonsteroidal anti-inflammatory drug. Cornea. 2006;25(10):1266-1267.

14. Kim SW, Ha BJ, Kim EK, Tchah H, Kim TI. The effect of topical bevacizumab on corneal neovascularization. Ophthalmology. 2008; 115(6): e33-e38.

15. Singh A, Naidu PS, Patil CS, Kulkarni SK. Effect of FK506 (tacrolimus) in animal models of inflammation. Inflammopharmacology. 2003; 11(3):249-257.

16. Ebihara N, Ohtomo K, Tokura T, Ushio H, Murakami A. Effect of tacrolimus on chemokine production by corneal myofibroblasts via toll-like receptors, compared with cyclosporine and dexamethasone. Cornea. 2011;30(6):702-708.

17. Turgut B, Guler M, Akpolat N, Demir T, Celiker U. The impact of tacrolimus on vascular endothelial growth factor in experimental corneal neovascularization. Curr Eye Res. 2011;36(1):34-40.
18. Park JH, Joo CK, Chung SK. Comparative study of tacrolimus and bevacizumab on corneal neovascularization in rabbits. Cornea. 2015; 34(4):449-455.

19. Yamazoe K, Yamazoe K, Yamaguchi T, Omoto M, Shimazaki J. Efficacy and safety of systemic tacrolimus in high-risk penetrating keratoplasty after graft failure with systemic cyclosporine. Cornea. 2014;33(11):1157-1163.

20. Abud TB, Amparo F, Saboo US, et al. A clinical trial comparing the safety and efficacy of topical tacrolimus versus methylprednisolone in ocular graft-versus-host disease. Ophthalmology. 2016;123(7):1449-1457.

21. Yuan J, Zhai JJ, Chen JQ, Ye CT, Zhou SY. Preparation of $0.05 \%$ FK506 suspension eyedrops and its pharmacokinetics after topical ocular administration. J Ocul Pharmacol Ther. 2009;25(4):345-350.

22. Yuan J, Zhai JJ, Huang X, Zhou SY, Chen JQ. Ocular safety and pharmacokinetics study of FK506 suspension eye drops after corneal transplantation. J Ocul Pharmacol Ther. 2012;28(2):153-158.

23. Türkcü FM, Cinar Y, Türkcü G, et al. Topical and subconjunctival ranibizumab (lucentis) for corneal neovascularization in experimental rat model. Cutan Ocul Toxicol. 2014;33(2):138-144.

24. Sonoda Y, Streilein JW. Impaired cell-mediated immunity in mice bearing healthy orthotopic corneal allografts. J Immunol. 1993;150(5): $1727-1734$.

25. Jianbin T, Liang H, Jufang $\mathrm{H}$, et al. Improved method of ink-gelatin perfusion for visualising rat retinal microvessels. Acta Histochem Cytochem. 2008;41(5):127-133.

26. Bakunowicz-Łazarczyk A, Urban B. Assessment of therapeutic options for reducing alkali burn-induced corneal neovascularization and inflammation. Adv Med Sci. 2016;61(1):101-112.

27. Lee P, Wang CC, Adamis AP. Ocular neovascularization: an epidemiologic review. Surv Ophthalmol. 1998;43(3):245-269.

28. Sawada S, Suzuki G, Kawase Y, Takaku F. Novel immunosuppressive agent, FK506. In vitro effects on the cloned T cell activation. J Immunol. 1987;139(6):1797-1803.

29. Kymionis GD, Klados NE, Kontadakis GA, Mikropoulos DG. Treatment of superior limbic keratoconjunctivitis with topical tacrolimus 0.03\% ointment. Cornea. 2013;32(11):1499-1501.

30. Shimmura-Tomita M, Shimmura S, Satake Y, et al. Keratoplasty postoperative treatment update. Cornea. 2013;32(Suppl 1):S60-S64.

31. Kim YG, Kamada N, Shaw MH, et al. The Nod2 sensor promotes intestinal pathogen eradication via the chemokine CCL2-dependent recruitment of inflammatory monocytes. Immunity. 2011;34(5):769-780.

32. Aomatsu T, Imaeda H, Takahashi K, et al. Tacrolimus (FK506) suppresses TNF- $\alpha$-induced CCL2 (MCP-1) and CXCL10 (IP-10) expression via the inhibition of $\mathrm{p} 38$ MAP kinase activation in human colonic myofibroblasts. Int J Mol Med. 2012;30(5):1152-1158.

33. Carmeliet $P$, Jain RK. Molecular mechanisms and clinical applications of angiogenesis. Nature. 2011;473(7347):298-307.

34. Cursiefen C, Rummelt C, Küchle M. Immunohistochemical localization of vascular endothelial growth factor, transforming growth factor alpha, and transforming growth factor beta 1 in human corneas with neovascularization. Cornea. 2000;19(4):526-533.

35. Ferrara N, Gerber HP, Lecouter J. The biology of VEGF and its receptors. Nat Med. 2003;9(6):669-676.

36. Zhong J, Deng Y, Tian B, et al. Hyaluronate acid-dependent protection and enhanced corneal wound healing against oxidative damage in corneal epithelial cells. J Ophthalmol. 2016;2016:6538051-10.

37. Bennett J, Cassidy H, Slattery C, Ryan MP, McMorrow T. Tacrolimus Modulates TGF- $\beta$ signaling to induce epithelial-mesenchymal transition in human renal proximal tubule epithelial cells. J Clin Med. 2016; $5(5): 50$.

38. Lan CC, Fang AH, Wu PH, Wu CS. Tacrolimus abrogates TGF- $\beta 1$ induced type I collagen production in normal human fibroblasts through suppressing p38MAPK signalling pathway: implications on treatment of chronic atopic dermatitis lesions. $J$ Eur Acad Dermatol Venereol. 2014;28(2):204-215. 


\section{Publish your work in this journal}

Drug Design, Development and Therapy is an international, peerreviewed open-access journal that spans the spectrum of drug design and development through to clinical applications. Clinical outcomes, patient safety, and programs for the development and effective, safe, and sustained use of medicines are the features of the journal, which has also been accepted for indexing on PubMed Central. The manuscript management system is completely online and includes a very quick and fair peer-review system, which is all easy to use. Visit http://www.dovepress.com/testimonials.php to read real quotes from published authors.

Submit your manuscript here: http://www.dovepress.com/drug-design-development-and-therapy-journal 